\title{
Inverse magnetic catalysis in QCD
}

\author{
Falk Bruckmann* \\ Institute for Theoretical Physics, Universität Regensburg \\ D-93040 Regensburg, Germany \\ E-mail: falk.bruckmann@physik.uni-regensburg.de
}

\section{Gergely Endrődi ${ }^{\dagger}$}

Institute for Theoretical Physics, Universität Regensburg

D-93040 Regensburg, Germany

E-mail: gergely.endrodiephysik.uni-regensburg.de

\section{Tamas G. Kovacs $\$$}

Institute of Nuclear Research of the Hungarian Academy of Sciences,

Bem tér 18/c, H-4026 Debrecen, Hungary

E-mail: kgteatomki.mta.hu

\begin{abstract}
We propose a physical mechanism for inverse magnetic catalysis, the suppression of the chiral condensate by an external magnetic field in QCD around the critical temperature. We show that this effect, seen in lattice simulations, is a result of how the sea quarks react to the magnetic field. We find that the suppression of the condensate happens because the quark determinant can suppress low quark modes by ordering the Polyakov loop. This mechanism is particularly efficient around $T_{c}$ where the Polyakov loop effective potential is flat and the determinant can have a significant ordering effect. Our picture suggests that for the description of QCD in large magnetic fields it is crucial to properly capture the interaction between the Polyakov loop and the sea quarks, both in low-energy effective models and on the lattice.
\end{abstract}

31 st International Symposium on Lattice Field Theory - LATTICE 2013

July 29 - August 3, 2013

Mainz, Germany

\footnotetext{
* Supported by the the Alexander von Humboldt foundation

${ }^{\dagger}$ Supported by the EU under grant number ITN STRONGnet 238353.

$\doteqdot$ Speaker.

§Supported by the Hungarian Academy of Sciences under "Lendület" grant No. LP2011-011 and by the EU Grant (FP7/2007-2013)/ERC No. 208740.
} 


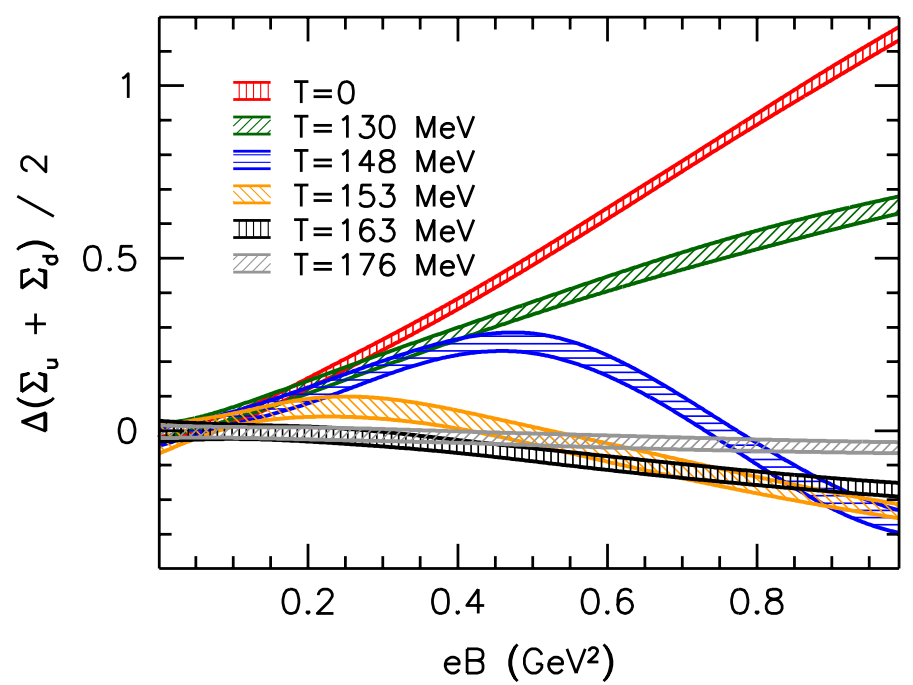

Figure 1: The dependence of the quark condensate on the magnetic field, computed at different temperatures around $T_{c}$.

\section{Introduction}

In heavy-ion collision experiments rapidly moving ions create large magnetic fields. There have been speculations how these large magnetic fields can influence the thermodynamics of these strongly interacting systems. For some time the common wisdom was that at any temperature the magnetic field should enhance the chiral condensate, an effect termed "magnetic catalysis" (see [1] for a review). This scenario was supported by calculations based on low-energy effective models and also by the first lattice QCD simulations. However these first lattice studies were based either on the quenched approximation [2] or they were performed using heavier than physical quarks and without a proper continuum extrapolation [3,4]. Later on, another lattice study, now using physical quark masses and performing an extrapolation to the continuum limit, reached a different conclusion [5]. There it was found that while at low temperature there is magnetic catalysis, around the crossover temperature, $T_{c}$, the reaction of the system to an external magnetic field is more complicated. The condensate is a non-monotonic function of the magnetic field and a large enough magnetic field actually suppresses the condensate rather than enhancing it. To illustrate this point, in Fig. 1 we show the dependence of the quark condensate on the magnetic field at various temperatures around $T_{c}$ (figure taken from Ref. [6]).

In the present work we offer a physical explanation of why this can happen. We show that there are two competing mechanisms through which the magnetic field influences the quark condensate. One involves valence quarks and that enhances the condensate, the other involves sea quarks and that suppresses the condensate. The non-monotonic behaviour observed around $T_{c}$ is a result of these two competing effects. We also identify the relevant gauge degrees of freedom playing the most important role in the sea quark suppression mechanism. These degrees of freedom are the Polyakov loops. We argue that effective low-energy models can account for this effect only if they properly take into account the interaction of sea quarks with the Polyakov loop. A more detailed account of this work can be found in Ref. [7]. 


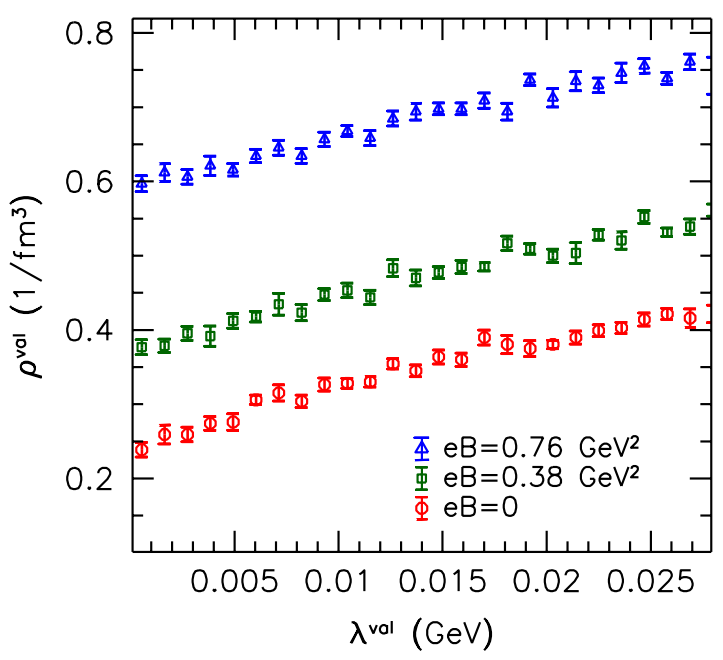

Figure 2: The spectral density of the staggered Dirac operator around zero computed with different magnetic fields in the Dirac operator. In all three cases the averaging was done over the same set of gauge configurations generated with zero magnetic field.

\section{Valence versus sea effect}

In order to understand how the external magnetic field influences the quark condensate, our starting point is the path integral expression for the condensate,

$$
\langle\bar{\psi} \psi\rangle(B)=\frac{1}{Z} \int d A \mathrm{e}^{-S(A)} \underbrace{\operatorname{det}[D(A, B)+m]}_{\text {"sea" }} \underbrace{\operatorname{Tr}\left[(D(A, B)+m)^{-1}\right]}_{\text {"valence" }},
$$

where the integration is over all the gauge field configurations. For illustration we use this schematic expression containing only one quark flavour of mass $m$; the generalisation to several flavours is straightforward. The dependence of the condensate on the external field, $B$, comes from two sources. Firstly, the quark determinant in the measure depends on $B$ which means that changing $B$ will change the relative weight of different gauge configuration. Secondly, the operator itself is also dependent on $B$, that is, the spectrum of the Dirac operator in a fixed gauge background changes with $B$. Using the terminology of Ref. [4] we call the first source of $B$ dependence "sea" effect and the second one "valence" effect.

Let us first look at the valence effect, how the spectrum of the Dirac operator in a fixed gauge background reacts when the magnetic field is switched on. In Figure 2 we show the spectral density of the staggered lattice Dirac operator around zero with three different values of the magnetic field in the Dirac operator. It is important to note that in all three cases the average spectral density was computed over the same set of gauge configurations, generated with zero magnetic field. Thus this figure demonstrates the valence effect only. We can easily see that the external magnetic field drives the low-lying Dirac modes closer to zero and thus enhances the spectral density. According to the Banks-Casher relation, for vanishing quark mass, the quark condensate is proportional to the spectral density of the Dirac operator at zero. By continuity we expect that if the quark mass is 


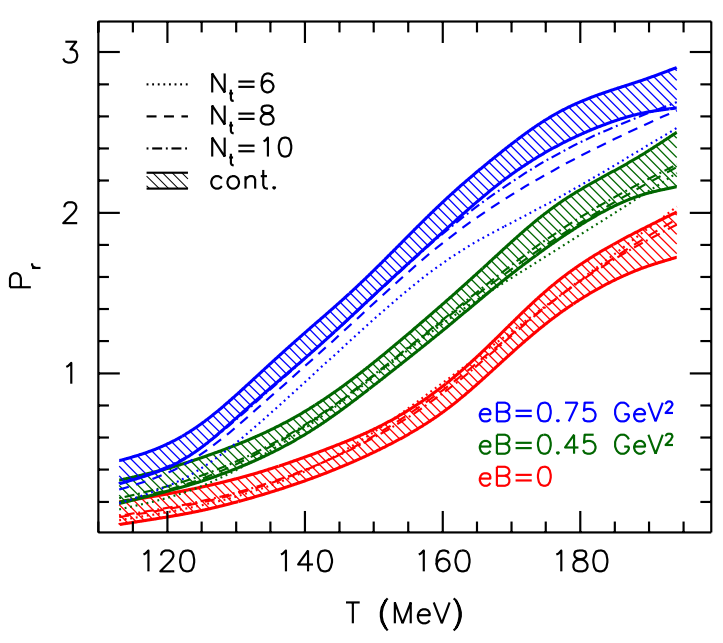

Figure 3: The renormalised average Polyakov loop as a function of the temperature for three different values of the background magnetic field. The different types of dashed line represent data obtained at different lattice spacings and the shaded regions correspond to the continuum extrapolations together with their uncertainties.

small, the condensate is still dominated by the lowest part of the spectrum. Thus an enhancement of low Dirac modes by the magnetic field implies and enhancement of the condensate. This is the basic mechanism behind magnetic catalysis happening well below $T_{c}$.

\subsection{Sea quarks and the Polyakov loop}

Let us now look at how the magnetic field in the quark determinant influences the condensate. Switching on the magnetic field will change the relative weight of the different gauge configurations. To see how this changes the typical gauge configurations contributing to the path integral we look for gauge field degrees of freedom that could play an important role in this mechanism. In Figure 3 we plot how the average Polyakov loop changes across the transition for three different values of the magnetic field. It is apparent from the figure that around the critical temperature the magnetic field strongly enhances the Polyakov loop.

To understand why the Polyakov loop is so strongly affected by the magnetic field we look at the quark action, the logarithm of the determinant,

$$
S_{q}=-\log \operatorname{det}(D+m)=-\sum_{i} \log \left(\lambda_{i}+m\right)
$$

where $\lambda_{i}$ are the eigenvalues of the Dirac operator. For small quark mass the fluctuations of this action are dominated by the small eigenvalues of the Dirac operator, so the determinant will strongly suppress those gauge configurations that have a large number of small Dirac modes. Since the magnetic field enhances small Dirac eigenvalues, switching on the magnetic field will amplify the suppression of small Dirac modes by the quark determinant.

How can we understand the connection of this effect to the Polyakov loop? How is the ordering of the Polyakov loop connected to the number of small Dirac modes? To see this we recall that 


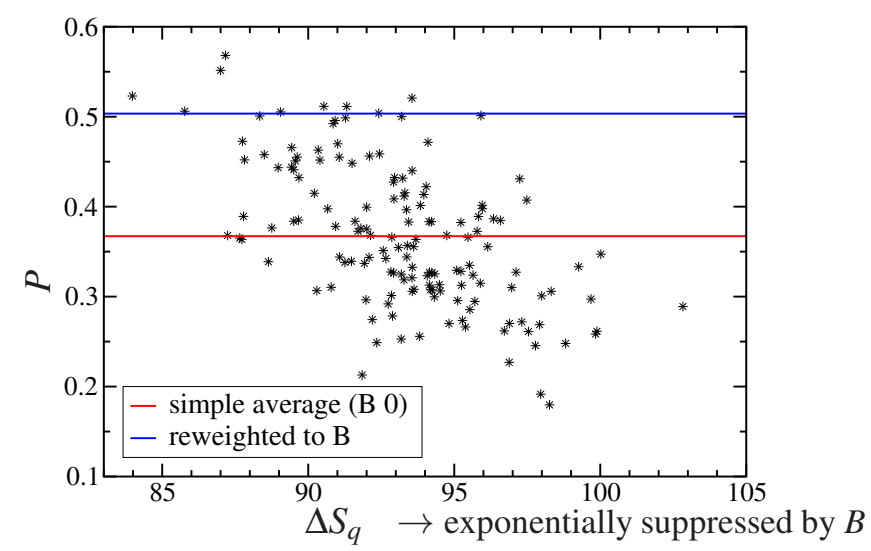

Figure 4: Scatter plot of the Polyakov loop versus the change in quark action, $\Delta S_{q}$, when the magnetic field is switched on. Each data point corresponds to a $10^{3} \times 4$ gauge configuration generated with zero magnetic field close to $T_{c}$. The lower (red) line indicates the simple average of the Polyakov loop for these configurations while the upper (blue) line shows the average computed by reweighting each term with the corresponding change in the quark effective action.

at low temperature, well below the transition, where the Polyakov loop is disordered, there are typically many small Dirac modes. This is how chiral symmetry is spontaneously broken there. In contrast, above $T_{c}$, chiral symmetry is restored and there are much less small Dirac modes. The physical reason for this is that above $T_{c}$ the Polyakov loop is ordered and the lowest quark modes are similar to the Matsubara modes with the corresponding eigenvalues being roughly proportional to the temperature. This is the mechanism behind chiral symmetry restoration above $T_{c}$.

In this way the quark action effectively works to order the Polyakov loop and restore chiral symmetry. Since the magnetic field enhances small Dirac modes, this ordering effect of sea quarks is also enhanced by the magnetic field. This means that configurations that have a small average Polyakov loop and as a result more small Dirac modes, get suppressed when the magnetic field is switched on. This is analogous to what happens in the presence of more quark flavours. Introducing more quark flavours will amplify the ordering effect of the determinant on the Polyakov loop and thus chiral symmetry is restored at a lower temperature. The magnetic field has a similar effect by pushing small Dirac modes down and enhancing the spectral density at the low end.

To demonstrate explicitly how this happens, in Fig. 4 we show a scatter plot of the Polyakov loop versus the change in quark action when the magnetic field is switched on,

$$
\Delta S_{q}=\log \operatorname{det}(D(0, A)+m)-\log \operatorname{det}(D(B, A)+m),
$$

for a set of gauge configurations generated with zero magnetic field. In the same figure we also show the simple average of the Polyakov loop over these gauge configurations (lower, thick red line) and the average computed by reweighting each configuration using the corresponding change in the quark action. The reweighted Polyakov loop is clearly much more ordered than the original one, in accordance with Fig. 3. 


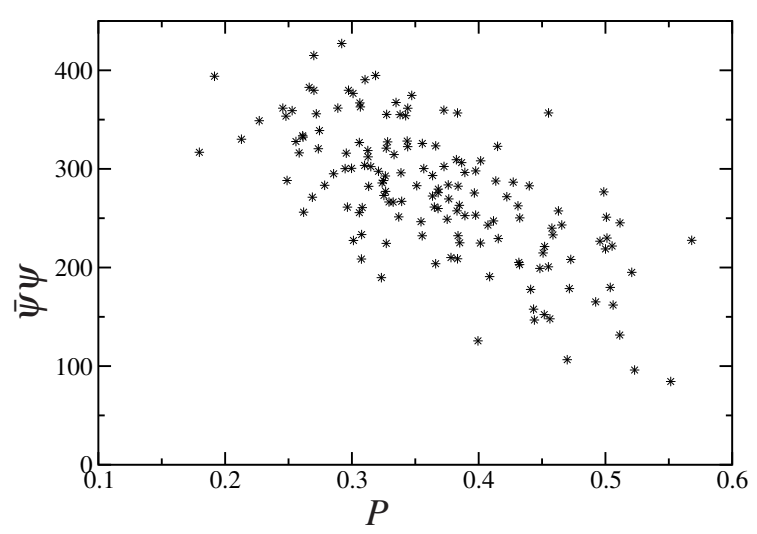

Figure 5: Scatter plot of the The Polyakov loop versus the quark condensate. Each data point corresponds to a $10^{3} \times 4$ gauge configuration generated with zero magnetic field close to $T_{c}$.

\section{The Polyakov loop and the condensate}

We saw that switching on the magnetic field orders the Polyakov loop and that implies a suppression of small quark modes by the determinant (sea). The very same small Dirac modes are the key to understand how the quark condensate is influenced by the magnetic field. To see this, we note that the condensate can be written as

$$
\bar{\psi} \psi=\operatorname{tr}(D+m)^{-1} \approx \sum \frac{1}{\lambda_{i}+m},
$$

where the sum is over the whole spectrum of the Dirac operator and $m$ is the quark mass. For small quark mass this sum is increasingly dominated by the lowest part of the Dirac spectrum. Thus the suppression of small Dirac modes by the magnetic field implies a suppression of the quark condensate.

To illustrate this point, in Fig. 5 we show a scatter plot of the quark condensate versus the Polyakov loop on the same set of configurations that we used for Fig. 4. The strong correlation between the condensate and the Polyakov loop indicates that these two quantities are both intimately connected to small quark modes as we described above.

\section{Competition between "sea" and "valence"}

We saw that when the magnetic field is switched on, sea quarks suppress those gauge configurations that have many small Dirac modes. At the same time the magnetic field in the operator enhances the condensate (valence). Both of these effects depend on how small quark modes react to the external magnetic field. The non-monotonic behaviour of the condensate with respect to the magnetic field can be explained by these two competing effects. Around the critical temperature, if the magnetic field is strong enough, the suppression of the condensate through the sea quarks wins, resulting in a condensate decreasing for larger fields.

It is curious why the sea suppression of the condensate can be so effective exactly around the critical temperature. The scenario that we described can also explain this. We saw that the most 
effective way of sea quarks to suppress small Dirac modes is to order the Polyakov loop. A small contribution from the magnetic field in the determinant to the Polyakov loop effective potential is usually not enough to have a significant ordering effect. This is true everywhere except around the critical temperature. Since at $T_{c}$ there is a cross-over, the minimum of the Polyakov loop effective potential there is very shallow and a small contribution to it from the magnetic field can have a significant ordering effect.

\section{Conclusions}

We described the mechanism that is responsible for inverse magnetic catalysis, the suppression of the quark condensate by an external magnetic field around $T_{c}$. We showed that the key to this is the effect of the magnetic field on small quark modes in the Dirac operator. The magnetic field enhances small quark modes which normally, through a Banks-Casher type argument, implies an enhancement of the condensate. This is what we called the valence effect. However, around $T_{c}$ the magnetic field in the determinant significantly alters the typical gauge configurations that contribute to the path integral. As a result, the Polyakov loop gets more ordered, small Dirac modes and also the condensate are suppressed. This is what we called sea effect.

Our picture also suggests the most important criteria that any effective model or lattice simulation has to fulfil in order to be able to properly account for inverse magnetic catalysis. Firstly, both in the quark determinant and in the operator measuring the condensate, the dominance of the lowest part of the Dirac spectrum is strongly dependent on the quark mass being small. Therefore, it is essential to use physical quark masses. Secondly, the sea effect depends on the interaction of the condensate and the Polyakov loop which is "mediated" by the small quark modes. Therefore, these degrees of freedom have to be included in some form in any low energy effective model used to describe how the magnetic field affects the condensate.

\section{References}

[1] I. A. Shovkovy, Lect. Notes Phys. 871 (2013) 13 [arXiv:1207.5081 [hep-ph]].

[2] P. V. Buividovich, M. N. Chernodub, E. V. Luschevskaya and M. I. Polikarpov, Phys. Lett. B 682 (2010) 484 [arXiv:0812.1740 [hep-lat]]; M. Muller-Preussker, B. Petersson and A. Schreiber, Phys. Rev. D 85 (2012) 114504 [arXiv:1203.3360 [hep-lat]].

[3] M. D’Elia, S. Mukherjee and F. Sanfilippo, Phys. Rev. D 82 (2010) 051501 [arXiv:1005.5365 [hep-lat]];

[4] M. D’Elia and F. Negro, Phys. Rev. D 83 (2011) 114028 [arXiv:1103.2080 [hep-lat]].

[5] G. S. Bali, F. Bruckmann, G. Endrodi, Z. Fodor, S. D. Katz, S. Krieg, A. Schafer and K. K. Szabo, JHEP 1202 (2012) 044 [arXiv:1111.4956 [hep-lat]].

[6] G. S. Bali, F. Bruckmann, G. Endrodi, Z. Fodor, S. D. Katz and A. Schafer, Phys. Rev. D 86 (2012) 071502 [arXiv:1206.4205 [hep-lat]].

[7] F. Bruckmann, G. Endrodi and T. G. Kovacs, JHEP 1304 (2013) 112 [arXiv:1303.3972 [hep-lat]]. 